\title{
ON THE COMMUTATOR LENGTHS OF CERTAIN CLASSES OF FINITELY PRESENTED GROUPS
}

\author{
H. DOOSTIE AND P. P. CAMPBELL
}

Received 23 June 2004; Revised 4 July 2005; Accepted 28 March 2006

For a finite group $G=\langle X\rangle(X \neq G)$, the least positive integer $\operatorname{ML}_{X}(G)$ is called the maximum length of $G$ with respect to the generating set $X$ if every element of $G$ may be represented as a product of at most $\mathrm{ML}_{X}(G)$ elements of $X$. The maximum length of $G$, denoted by $\operatorname{ML}(G)$, is defined to be the minimum of $\left\{\operatorname{ML}_{X}(G) \mid G=\langle X\rangle, X \neq G, X \neq\right.$ $\left.G-\left\{1_{G}\right\}\right\}$. The well-known commutator length of a group $G$, denoted by $c(G)$, satisfies the inequality $c(G) \leq \operatorname{ML}\left(G^{\prime}\right)$, where $G^{\prime}$ is the derived subgroup of $G$. In this paper we study the properties of $\operatorname{ML}(G)$ and by using this inequality we give upper bounds for the commutator lengths of certain classes of finite groups. In some cases these upper bounds involve the interesting sequences of Fibonacci and Lucas numbers.

Copyright (c) 2006 Hindawi Publishing Corporation. All rights reserved.

\section{Introduction}

For an abstract group $G$ the commutator length $c(G)$ is defined to be $c(G)=\operatorname{Sup}\{\lambda(g)$ | $\left.g \in G^{\prime}\right\}$, where $\lambda(g)$ is the minimal number of commutators of which $g$ is the product. This notion has been studied by many authors during the years and the results of the combinatorial methods estimate or calculate the commutator lengths of abstract groups which are mainly infinite (one may see $[2,6-10]$, e.g.). For a finite group $G=\langle X\rangle$ we examine the effect of the generating set on the evaluation of this number by considering the following definitions, this gives us a method to calculate upper bounds for $c(G)$. Let $G=\langle X\rangle(X \neq G)$ be a finite group. Then the following hold.

Definition 1.1. $\operatorname{ML}_{X}(G)$, the maximum length of $G$ relative to the generating set $X$, is defined to be $\max \{\lambda(g) \mid g \in G\}$, where $\lambda(g)$ is the minimum number of the elements of $X$ of which $g$ is the product.

Definition 1.2. The maximum length of a group $G$, denoted by $\operatorname{ML}(G)$, is defined to be the minimum of all numbers $\operatorname{ML}_{X}(G)$, for all generating sets $X(X \neq G)$ of $G$.

Our notations are fairly standard, we use $[x]$ for the integer part of the real number $x,[a, b]=a^{-1} b^{-1} a b$ is defined to be the commutator of the elements $a$ and $b$ of a group, 
the usual notation $N \times{ }_{\varphi} H$ is used for the semidirect product of the group $N$ by $H$, where $\varphi: H \rightarrow \operatorname{Aut}(N)$ is a homomorphism such that $h \varphi=\varphi_{h}$ and $\varphi_{h}: N \rightarrow N$ is an element of $\operatorname{Aut}(N)$, and the Reidemeister-Schreier algorithm in the form given in [1] will be used to find presentations of subgroups. In the following sections we study certain classes of finite groups for their maximal lengths and find upper bounds for the commutator lengths. The groups studied here are the dihedral groups $D_{2 n}=\left\langle a, b \mid a^{2}=b^{n}=(a b)^{2}=1\right\rangle, n \geq 3$, the quaternion groups $Q_{2^{n}}=\left\langle a, b \mid a^{2^{n-1}}=1, b^{2}=a^{2^{n-2}}, b^{-1} a b=a^{-1}\right\rangle, n \geq 3$, the semidirect products $D_{2 n} \times{ }_{\varphi} Z_{2 m}$, and $Q_{2^{n}} \times{ }_{\varphi} Z_{2 m},\left(n, m \geq 3\right.$ ) (where if $Z_{2 m}=\langle c\rangle$, then $\varphi: Z_{2 m} \rightarrow$ $\operatorname{Aut}\left(D_{2 n}\right)$ is such that $c \varphi=\varphi_{c}$; and $\varphi_{c}: D_{2 n} \rightarrow D_{2 n}$ is defined by $a \varphi_{c}=a$ and $b \varphi_{c}=b^{-1}$, a similar $\varphi$ exists for $\left.Q_{2^{n}} \times{ }_{\varphi} Z_{2 m}\right)$, the direct products $D_{2 n} \times Z_{2 m}$ and $Q_{2^{n}} \times Z_{2 m}$, and the following classes of groups:

$$
\begin{gathered}
G_{1}=\left\langle a, b \mid a^{2}=b^{n}=a b a b^{-1} a b^{2} a b^{-1} a b^{-2} a b=1\right\rangle, \quad n \geq 3, \\
G_{2}=\left\langle a, b \mid a^{2}=b^{n},\left(a b^{2}\right)^{2}\left(a b^{-1}\right)^{2}=b^{2 n}\right\rangle, \quad n \neq 0, \\
G_{3}=\left\langle a, b \mid a^{2}=b^{n}=1, a b a b^{-2} a b^{3} a b^{-2} a b^{-1} a b=1\right\rangle, \quad n \geq 3, \\
G_{4}=\left\langle a, b \mid a^{2}=b^{5}=1,[a, b]^{k}\left[a, b^{3}\right]=1\right\rangle, \quad k \geq 1 .
\end{gathered}
$$

The groups $G_{i}(i=1,2,3,4)$ are soluble and have been studied for their structures and orders in [3-5]. These groups are generalizations of the well-known Coxeter groups.

We will use the Fibonacci and Lucas numbers:

$$
\begin{gathered}
f_{0}=f_{1}=1, \quad f_{n}=f_{n-1}+f_{n-2}, \quad n \geq 2, \\
g_{0}=2, \quad g_{1}=1, \quad g_{n}=g_{n-1}+g_{n-2}, \quad n \geq 2,
\end{gathered}
$$

which are related to each other via the relation $g_{n}=f_{n-2}+f_{n}$. In Section 2 we study the notion of the maximum length for the mentioned direct and semidirect products, and by using the inequality $c(G) \leq \operatorname{ML}\left(G^{\prime}\right)$ which holds for every nonabelian finite group $G$, we give upper bounds for the commutator lengths of the groups $G_{i}(i=1,2,3,4)$, in Sections 3 and 4 .

2. The groups $D_{2 n}, Q_{2^{n}}, D_{2 n} \times Z_{2 m}, Q_{2^{n}} \times Z_{2 m}, D_{2 n} \times{ }_{\varphi} Z_{2 m}, Q_{2^{n}} \times{ }_{\varphi} Z_{2 m}$

Let $m, n \geq 3$ be integers. By the definitions of the direct and semidirect products, we get the following presentations:

$$
\begin{gathered}
D_{2 n} \times Z_{2 m}=\left\langle a, b, c \mid a^{2}=b^{n}=(a b)^{2}=c^{2 m}=[a, c]=[b, c]=1\right\rangle, \\
Q_{2^{n}} \times Z_{2 m}=\left\langle a, b, c \mid a^{2^{n-1}}=1, b^{2}=a^{2^{n-2}}, b^{-1} a b a=c^{2 m}=[a, c]=[b, c]=1\right\rangle, \\
D_{2 n} \times Z_{2 m}=\left\langle a, b, c \mid a^{2}=b^{n}=(a b)^{2}=c^{2 m}=1, c^{-1} a c a=1, c^{-1} b c b=1\right\rangle, \\
Q_{2^{n}} \times_{\varphi} Z_{2 m}=\left\langle a, b, c \mid a^{2^{n-1}}=1, b^{2}=a^{2^{n-2}}, b^{-1} a b a=c^{2 m}=1, c^{-1} a c a=1, c^{-1} b c b=1\right\rangle,
\end{gathered}
$$

where $\varphi$ is the homomorphism defined in the last section. 
As an immediate result of the definitions we have the following.

Lemma 2.1. For every finite groups $G_{1}=\langle X \mid R\rangle$ and $G_{2}=\langle Y \mid S\rangle$,

$$
\operatorname{ML}_{\{X, Y\}}\left(G_{1} \times G_{2}\right) \leq \operatorname{ML}_{X}\left(G_{1}\right)+\operatorname{ML}_{Y}\left(G_{2}\right) .
$$

Proof. Obviously, $G_{1} \times G_{2}=\langle X, Y \mid R, S,[X, Y]\rangle$, where $[X, Y]=\{[x, y] \mid x \in X, y \in Y\}$. There exists an element $g \in G_{1} \times G_{2}$ such that $\operatorname{ML}_{\{X, Y\}}\left(G_{1} \times G_{2}\right)=\lambda(g)$, and $g=$ $\left(x_{1} x_{2} \cdots x_{m}\right)\left(y_{1} y_{2} \cdots y_{n}\right)$, where $x_{i} \in X$ and $y_{i} \in Y$ (for $\left[x_{i}, y_{j}\right]=1$ holds for every $i$ and $j)$. Let $g_{1}=x_{1} x_{2} \cdots x_{m}$ and $g_{2}=y_{1} y_{2} \cdots y_{n}$. The definition of $\lambda(g)$ then yields

$$
\operatorname{ML}_{\{X, Y\}}\left(G_{1} \times G_{2}\right)=\lambda(g)=m+n \leq \lambda\left(g_{1}\right)+\lambda\left(g_{2}\right) \leq \operatorname{ML}_{X}\left(G_{1}\right)+\operatorname{ML}_{Y}\left(G_{2}\right) .
$$

Proposition 2.2. $m, n \geq 3$ are integers, then

(i) for a nonabelian metacyclic group $G, c(G) \leq\left|G^{\prime}\right|-1$;

(ii) for a metabelian group $G$, where $\left|G^{\prime}\right|=p_{1}^{\alpha_{1}} p_{2}^{\alpha_{2}} \cdots p_{k}^{\alpha_{k}}$ (here $p_{1}, p_{2}, \ldots$ are different primes $) c(G) \leq\left(p_{1}^{\alpha_{1}}+\cdots+p_{k}^{\alpha_{k}}\right)-k$;

(iii) $\operatorname{ML}\left(D_{2 n}\right) \leq[n / 2]+1$ and $\operatorname{ML}\left(Q_{2^{n}}\right) \leq 2^{n-3}+3$;

(iv) $\operatorname{ML}\left(D_{2 n} \times Z_{2 m}\right) \leq[n / 2]+2 m$ and $\operatorname{ML}\left(Q_{2^{n}} \times Z_{2 m}\right) \leq 2^{n-3}+2 m+2$;

(v) $\operatorname{ML}\left(D_{2 n} \times_{\varphi} Z_{2 m}\right) \leq[n / 2]+2 m$;

(vi) $\operatorname{ML}\left(Q_{2^{n}} \times{ }_{\varphi} Z_{2 m}\right) \leq 2^{n-3}+2 m$.

Proof. For a nonabelian metacyclic group $G$, the derived subgroup $G^{\prime}$ is a cyclic group, so $\operatorname{ML}\left(G^{\prime}\right)=\left|G^{\prime}\right|-1$ and (i) follows at once.

For a metabelian group $G$, where $\left|G^{\prime}\right|=p_{1}^{\alpha_{1}} p_{2}^{\alpha_{2}} \cdots p_{k}^{\alpha_{k}}$, we may use the direct decomposition of $G^{\prime}$ to get (ii).

We give proof of the first part of (iii), the second part is similar. The elements of $D_{2 n}$ are of the form $a^{i} b^{j}$, where $i=0,1$ and $j=0,1, \ldots, n-1$. Consider two cases for $n$ and for every $g \in D_{2 n}$ compute $\lambda(g)$, the minimum number of the elements of $X=\langle a, b\rangle$ of which $g$ is the product. We see that the relations $(a b)^{2}=a^{2}=b^{n}=1$ of $D_{2 n}$ yield the relations $b^{n-k}=b^{-k}=a b^{k} a$, for every integer $k$.

Case 1. $n$ is even. It is easy to see that

$$
\lambda\left(b^{n-k}\right)= \begin{cases}k+2 & \text { if } 1 \leq k \leq \frac{n}{2}-1, \\ n-k & \text { if } \frac{n}{2} \leq k \leq n-1 .\end{cases}
$$

Also,

$$
\lambda\left(a b^{k}\right)= \begin{cases}k+1 & \text { if } 1 \leq k \leq \frac{n}{2}, \\ n-k+1 & \text { if } \frac{n}{2}+1 \leq k \leq n-1 .\end{cases}
$$

So, in this case $\operatorname{ML}_{X}\left(D_{2 n}\right)=\max \left\{\lambda(g) \mid g \in D_{2 n}\right\}=n / 2+1$. 
4 On the commutator lengths of certain classes of groups

Case 2. $n$ is odd. In this case similar classification of the elements gives us

$$
\lambda\left(b^{n-k}\right)= \begin{cases}k+2 & \text { if } 1 \leq k \leq \frac{n-3}{2}, \\ n-k & \text { if } \frac{n-1}{2} \leq k \leq n-1 .\end{cases}
$$

Also,

$$
\lambda\left(a b^{k}\right)= \begin{cases}k+1 & \text { if } 1 \leq k \leq \frac{n-1}{2}, \\ n-k+1 & \text { if } \frac{n+1}{2} \leq k \leq n-1,\end{cases}
$$

and in this case $\operatorname{ML}_{X}\left(D_{2 n}\right)=\max \left\{\lambda(g) \mid g \in D_{2 n}\right\}=(n+1) / 2$. Consequently, for every $n \geq 3, \operatorname{ML}\left(D_{2 n}\right) \leq \operatorname{ML}_{X}\left(D_{2 n}\right)=[n / 2]+1$. A similar proof for $Q_{2^{n}}$ yields $\operatorname{ML}_{X}\left(Q_{2^{n}}\right)=$ $2^{n-3}+3$, where $X=\langle a, b\rangle$ is the generating set of $Q_{2^{n}}$, and $\operatorname{ML}\left(Q_{2^{n}}\right) \leq \operatorname{ML}_{X}\left(Q_{2^{n}}\right)=2^{n-3}+$ 3.

The inequalities of (iv) are the results of Lemma 2.1 and the calculations of part (iii), where we know that $\operatorname{ML}_{\{a, b\}}\left(D_{2 n}\right)=[n / 2]+1, \operatorname{ML}_{\{a, b\}}\left(Q_{2^{n}}\right)=2^{n-3}+3$, and $\operatorname{ML}_{\{c\}}\left(Z_{2 m}\right)=$ $2 m-1$.

To prove (v) we see that up to the relations $c a=a c, c b=b^{-1} c$, and $b a=a b^{-1}$ of the group $D_{2 n} \times{ }_{\varphi} Z_{2 m}$ the $4 m n$ elements of this group may be considered as the union of the following sets:

$$
\begin{gathered}
S_{1}=\left\{c^{i} \mid 1 \leq i \leq 2 m-1\right\}, \\
S_{2}=\left\{a^{i} b^{j} \mid 0 \leq i \leq 1,0 \leq j \leq n-1\right\}, \\
S_{3}=\left\{a c^{i} \mid 1 \leq i \leq 2 m-1\right\}, \\
S_{4}=\left\{b^{i} c^{j} \mid 1 \leq i \leq n-1,1 \leq j \leq 2 m-1\right\}, \\
S_{5}=\left\{a b^{i} c^{j} \mid 1 \leq i \leq n-1,1 \leq j \leq 2 m-1\right\} .
\end{gathered}
$$

Since the relations $b^{n-k}=a b^{k} a, b^{n-k}=c b^{k} c$, and $c^{k}=a c^{k} a$ hold in the group, for every integer $k$, then the minimum length $\lambda(g)$ is definitely acceptable for every $g \in D_{2 n} \times_{\varphi} Z_{2 m}$, in the similar way as in (iii) and we get

$$
\begin{gathered}
\max \left\{\lambda(g) \mid g \in S_{1}\right\}=2 m-1, \\
\max \left\{\lambda(g) \mid g \in S_{2}\right\}=1+\left[\frac{n}{2}\right], \\
\max \left\{\lambda(g) \mid g \in S_{3}\right\}=2 m, \\
\max \left\{\lambda(g) \mid g \in S_{4}\right\}=\left[\frac{n}{2}\right]+2 m-1, \\
\max \left\{\lambda(g) \mid g \in S_{5}\right\}=\left[\frac{n}{2}\right]+2 m .
\end{gathered}
$$

Consequently, $\operatorname{ML}\left(D_{2 n} \times{ }_{\varphi} Z_{2 m}\right) \leq \operatorname{ML}_{\{a, b, c\}}\left(D_{2 n} \times{ }_{\varphi} Z_{2 m}\right)=[n / 2]+2 m$. 
The proof of (vi) is similar to the above proof and one may get the result by considering the $m 2^{n+1}$ elements of the group $Q_{2^{n}} \times_{\varphi} Z_{2 m}$ as the union of the following sets:

$$
\begin{gathered}
S_{1}=\left\{a^{i} b^{j} \mid 0 \leq i \leq 2^{n-1}-1,0 \leq j \leq 1\right\}, \\
S_{2}=\left\{a^{i} c^{j} \mid 0 \leq i \leq 2^{n-1}-1,1 \leq j \leq 2 m-1\right\}, \\
S_{3}=\left\{b c^{i} \mid 1 \leq i \leq 2 m-1\right\}, \\
S_{4}=\left\{a^{i} b c^{j} \mid 1 \leq i \leq 2^{n-1}-1,1 \leq j \leq 2 m-1\right\} .
\end{gathered}
$$

And this completes the proof.

\section{The groups $G_{1}$ and $G_{2}$}

The groups $G_{1}$ and $G_{2}$ are finite and nonmetabelian soluble groups for many values of $n$ (see $[4,5])$. The following propositions are our main results on the commutator lengths of these groups.

Proposition 3.1. For every $n \geq 3$, where g.c.d $(n, 3) \neq 1$,

$$
c\left(G_{1}\right) \leq \begin{cases}g_{n} & \text { if } n \equiv \pm 3(\bmod 12), \\ 4 f_{n / 2-1} & \text { if } n \equiv 0(\bmod 12), \\ 2 g_{n / 2} & \text { if } n \equiv 6(\bmod 12) .\end{cases}
$$

Proposition 3.2. For every $n \geq 4$,

$$
c\left(G_{2}\right) \leq \begin{cases}2 n-1 & \text { if } n \equiv \pm 1(\bmod 3), \\ \frac{n+7}{2} & \text { if } n \equiv 3(\bmod 6), \\ \frac{n+8}{2} & \text { if } n \equiv 0(\bmod 6) .\end{cases}
$$

Proof of Proposition 3.1. Let $n \equiv \pm 3(\bmod 12)$. Using the results of [5] gives us here the following presentation for $G_{1}^{\prime}$ :

$$
G_{1}^{\prime}=\left\langle x, y \mid(x y)^{2}=(y x)^{2},\left[x^{2}, y\right]=\left[y^{2}, x\right]=1, x y=y^{-f_{n-2}} x^{f_{n-3}}, x^{1+f_{n-2}}=y^{f_{n-1}}\right\rangle .
$$

First we show that the relations $y^{g_{n}}=x^{g_{n}}=1$ hold in $G_{1}^{\prime}$.

The relation $x y=y^{-f_{n-2}} x^{f_{n-3}}$ gives us

$$
x y^{f_{n-1}} x^{-1}=y^{-f_{n-2} f_{n-3}} x^{\left(-1+f_{n-3}\right) f_{n-1}} .
$$

For $f_{n-3}$ is odd, then $\left[y^{-f_{n-2}}, x^{-1+f_{n-3}}\right]=1$. This relation together with the last relation of $G_{1}^{\prime}$ yields

$$
y^{f_{n-1}}=y^{-f_{n-2} f_{n-3}} x^{\left(-1+f_{n-3}\right) f_{n-1}} .
$$


6 On the commutator lengths of certain classes of groups

And substituting for $y^{f_{n-1}}$ yields

$$
x^{\left(1+f_{n-2}\right)^{2}-f_{n-1}\left(-1+f_{n-3}\right)}=1 .
$$

By an inductive method we may show that $g_{n}=\left(1+f_{n-2}\right)^{2}-f_{n-1}\left(-1+f_{n-3}\right)$ and then $x^{g_{n}}=1$. A similar proof exists for $y^{g_{n}}=1$ and since $G_{1}^{\prime}$ is of order $2 g_{n}$ (see [5]), $g_{n}$ is the order of $y$.

Now consider the relations $\left[x^{2}, y\right]=\left[y^{2}, x\right]=1$ and conclude that the words $x y^{-1+g_{n}}$ (or $y^{-1+g_{n}} x$ ) are the words of largest length; that is, $c\left(G_{1}\right) \leq \operatorname{ML}\left(G_{1}^{\prime}\right) \leq \operatorname{ML}_{\{x, y\}}\left(G_{1}^{\prime}\right)=g_{n}$.

Let $n \equiv 6(\bmod 12)$. In this case $G_{1}^{\prime}$ may be presented as

$$
\left\langle x, y \mid(x y)^{2}=(y x)^{2},\left[x^{2}, y\right]=\left[y^{2}, x\right]=1, x^{1+f_{n-3}} y^{-1+f_{n-2}}, x^{-1+f_{n-2}}=y^{f_{n-1}}\right\rangle .
$$

This group is of order $2\left(-2+g_{n}\right)$ (see [5]). First we show that the relations $x^{g_{n / 2}}=y^{g_{n / 2}}=$ 1 hold in $G_{1}^{\prime}$. Combining the last two relations of $G_{1}^{\prime}$ yields $x^{f_{n-4}-f_{2}}=y^{f_{n-3}+f_{1}}$. Again combining this relation with $x^{1+f_{n-3}}=y^{-1+f_{n-2}}$ gives us $x^{f_{n-5}+f_{3}}=y^{f_{n-4}-f_{2}}$. We repeat this method and get the relations

$$
x^{f_{n-2-i}-(-1)^{i} f_{i}}=y^{f_{n-1-i}+(-1)^{i} f_{i-1}}, \quad i=2,3, \ldots
$$

For $i=(n-4) / 2$ and $i=(n-6) / 2$ we get $x^{t}=y^{t}$ and $x^{t}=y^{2 t}$, respectively, where $t=$ $f_{n / 2}+f_{(n-4) / 2}$. Consequently, $y^{t}=1$ holds in $G_{1}^{\prime}$. Now it is easy to see that $t=g_{n / 2}$. The word $y x^{-1+g_{n / 2}} y^{-1+g_{n / 2}} x$ is one of the words of maximum length. So $c\left(G_{1}\right) \leq \operatorname{ML}\left(G_{1}^{\prime}\right) \leq$ $\operatorname{ML}_{\{x, y\}}\left(G_{1}^{\prime}\right)=2 g_{n / 2}$ as required in this case.

The remained case is $n \equiv 0(\bmod 12)$. In this case $G_{1}^{\prime}$ has the previous case's presentation. We first show that the relations

$$
x^{f_{n / 2-1}}=y^{3 f_{n / 2-1}}, \quad y^{5 f_{n / 2-1}}=1
$$

hold in $G_{1}^{\prime}$. As well as in the last case we get the relations

$$
x^{f_{n-2-i}-(-1)^{i} f_{i}}=y^{f_{n-1-i}+(-1)^{i} f_{i-1}}, \quad i=2,3, \ldots
$$

If we let $i=-2+n / 2$ then, after a simplification we get

$$
x^{f_{n / 2-1}}=y^{3 f_{n / 2-1}}
$$

Raising both sides to the power 3 yields $x^{3 f_{n / 2-1}}=y^{9 f_{n / 2-1}}$. Also for the value $i=-3+n / 2$ we get $x^{3 f_{n / 2-1}}=y^{4 f_{n / 2-1}}$. Consequently, $y^{5 f_{n / 2-1}}=1$, and these relations together with the relations $\left[x^{2}, y\right]=\left[y^{2}, x\right]=1$ show that the word

$$
y^{-1+5 f_{n / 2-1}} x^{-1+f_{n / 2-1}} y x
$$


is of the largest length in $G_{1}^{\prime}$. However, by considering the relation $x^{f_{n / 2-1}}=y^{3 f_{n / 2-1}}$, this word will be reduced to

$$
y^{-1+2 f_{n / 2-1}} x^{-1+2 f_{n / 2-1}} y x
$$

then $c\left(G_{1}\right) \leq \operatorname{ML}\left(G_{1}^{\prime}\right) \leq \operatorname{ML}_{\{x, y\}}\left(G_{1}^{\prime}\right)=4 f_{n / 2-1}$, as required. This completes the proof.

Proof of Proposition 3.2. For every $n \equiv \pm 1(\bmod 3), G_{2}$ is a metabelian group and as a result of the computations of [4], $G_{2}^{\prime}$ is a cyclic group of order $2 n$. So $c\left(G_{2}\right) \leq 2 n-1$ comes from the results of Section 2.

Let $n \equiv 3(\bmod 6)$. Using the Todd-Coxeter coset enumeration algorithm gives us the presentation

$$
G_{2}^{\prime}=\left\langle x, y \mid(x y)^{2}=y^{n},(y x)^{2}=x^{n}, x^{3} y^{3}=1\right\rangle .
$$

It is easy to show the validity of the relations $x^{n}=y^{n}$ and $x^{2 n}=y^{2 n}=1 \mathrm{in} G_{2}^{\prime}$. Let $n=6 k+3$. We claim that the words $w_{1}=x^{2} y^{3 k+1} x y^{2}$ and $w_{2}=y^{2} x^{3 k+1} y x^{2}$ in $G_{1}^{\prime}$ are of the largest length $3 k+5$. Indeed, the relations $x^{3} y^{3}=1, x y x=y^{n-1}$, and $y x y=x^{n-1}$ show that any word with maximal length could not contain the subwords $x^{2} y^{2}$ and $y^{2} x^{2}$. The remained words which have to be examined are indeed $w_{3}=y^{3 k+1} x y^{2} x$ and $w_{4}=x^{3 k+1} y x^{2} y$. These words are of length $3 k+4$, for we have

$$
w_{3}=y^{3 k}(y x y) y x=y^{3 k} x^{6 k+2} y x=x^{3 k+2} y x .
$$

Similarly, $w_{4}$ is of length $3 k+4$. To complete the proof we now show that $w_{1}$ and $w_{2}$ are of length $3 k+5$. By using the relations we get

$$
w_{1}=x^{2} y^{3 k}(y x y) y=x^{2} y^{3 k} x^{6 k+2} y=x^{2}\left(y^{3 k} x^{3 k}\right) x^{3 k+2} y=x^{3 k+4} y,
$$

and in a similar way, $w_{2}$ will be reduced to $y^{3 k+4} x$. So $c\left(G_{2}\right) \leq \operatorname{ML}\left(G_{2}^{\prime}\right) \leq \operatorname{ML}_{\{x, y\}}\left(G_{2}^{\prime}\right)=$ $3 k+5$.

Let $n \equiv 0(\bmod 6)$. We use the Todd-Coxeter coset enumeration algorithm to find a presentation for $G_{2}^{\prime}$. In two different cases, $n \equiv 0(\bmod 12)$ and $n \equiv 6(\bmod 12)$, we get the following presentations:

$$
\begin{aligned}
& G_{2}^{\prime}=\left\langle x, y \mid\left[x^{2}, y\right]=\left[x, y^{3}\right]=x^{4}=y^{n}=R_{1}=R_{2}=1,(x y)^{n / 2}=x^{2}\right\rangle, \\
& G_{2}^{\prime}=\left\langle x, y \mid\left[x^{2}, y\right]=\left[x, y^{3}\right]=x^{4}=y^{n}=R_{1}=R_{2}=1,(x y)^{n / 2}=y^{n / 2}\right\rangle,
\end{aligned}
$$

respectively, where $R_{1}=y^{2+n / 2} x^{3} y^{-1} x y^{-1} x^{3}$ and $R_{2}=y x y^{-1} x y x^{3} y^{-1} x$. The largest power of $x$ in every word of $G_{2}^{\prime}$ is equal to 3 , however, the largest power of $y$ is $n / 2+1$, for $R_{1}=1$ yields

$$
y^{2+n / 2}=x y x^{3} y x
$$

This relation also gives us the relation $(x y)^{2}=y^{2+n / 2} x$. The relation $R_{2}=1$ yields $y x y^{n-1} x y$ $=x^{3} y x$ and hereby we deduce that the only words of maximum length must be among 
8 On the commutator lengths of certain classes of groups

the following words:

$$
w_{1}=x^{3} y^{1+n / 2}, \quad w_{2}=y^{1+n / 2} x^{3}, \quad w_{3}=x^{3} y^{1+n / 2} x y, \quad w_{4}=x y x^{3} y^{1+n / 2} .
$$

Obviously the words $w_{1}$ and $w_{2}$ are of length $4+n / 2$, however $w_{4}$ is of length 5 , for we see that

$$
w_{4}=\left(x y x^{3} y\right) y^{n / 2}=\left(y^{2+n / 2} x^{-1}\right) y^{n / 2}=y^{2+n / 2} x^{3} y^{n / 2}=y^{n+2} x^{3}=y^{2} x^{3} .
$$

Finally $w_{3}$ is also of length $4+n / 2$, for

$$
w_{3}=y^{n / 2}\left(x^{3} y x\right) y=y^{n / 2}\left(y x y^{n-1} x y\right) y=y^{n}\left(y x y^{-1+n / 2} x y^{2}\right)=y x y^{-1+n / 2} x y^{2} .
$$

Consequently, $c\left(G_{2}\right) \leq \operatorname{ML}\left(G_{2}^{\prime}\right) \leq \operatorname{ML}_{\{x, y\}}\left(G_{2}^{\prime}\right)=4+n / 2$. This completes the proof.

\section{The groups $G_{3}$ and $G_{4}$}

These groups are examples of metacyclic groups and we have the following result.

Proposition 4.1. For every $n \geq 3$, the groups $G_{3}$, and for every $k \geq 1$, the groups $G_{4}$ are finite. Moreover, $c\left(G_{3}\right) \leq\left(2^{n}-3-(-1)^{n}\right) / 3$ and $c\left(G_{4}\right) \leq k\left(k^{3}+2 k^{2}+4 k+3\right)$.

Proof. The subgroup $H=\left\langle x_{i}=\left[a^{-1}, b^{-1}\right]^{b^{-i}}: 0 \leq i \leq n-1\right\rangle$ of $G_{3}$ is indeed the derived subgroup of $G_{3}$ (one may easily check that $\left|G_{3}: H\right|=\left|G_{3}: G_{3}^{\prime}\right|=2 n$ and $H \subseteq G_{3}^{\prime}$ ). A presentation for $H$ may be given as

$$
H=\left\langle x_{0}, \ldots, x_{n-1} \mid x_{i} x_{i+1}^{2}=1, x_{n-1} x_{0}^{2}=1,0 \leq i \leq n-2\right\rangle .
$$

Obviously this group is finite and cyclic of order $\left(2^{n}-(-1)^{n}\right) / 3$, and $c\left(G_{3}\right) \leq \operatorname{ML}_{\{x, y\}}\left(G_{3}^{\prime}\right)=$ $\left|G_{3}^{\prime}\right|-1=\left(2^{n}-3-(-1)^{n}\right) / 3$ is a result of Section 2 . In a similar way we consider the subgroup

$$
K=\left\langle\left[a^{-1}, b\right],\left[b^{-1}, a\right]^{b^{-i}}: 0 \leq i \leq 3\right\rangle
$$

of $G_{4} . K$ is the derived subgroup of $G_{4}$ and may be presented by

$$
K=\left\langle a_{1}, \ldots, a_{5} \mid a_{1} a_{2} a_{3} a_{4} a_{5}=1, a_{i}^{k}=a_{i+1} a_{i+2}, 1 \leq i \leq 5\right\rangle,
$$

(where indices are reduced modulo 5). An almost easy simplification of the relations shows that $K$ is a cyclic group of order $k^{4}+2 k^{3}+4 k^{2}+3 k+1$, and $c\left(G_{4}\right) \leq \operatorname{ML}_{\{x, y\}}\left(G_{4}^{\prime}\right)=$ $\left|G_{4}^{\prime}\right|-1=k\left(k^{3}+2 k^{2}+4 k+3\right)$ is a result of Section 2. This completes the proof.

\section{References}

[1] M. J. Beetham and C. M. Campbell, A note on the Todd-Coxeter coset enumeration algorithm, Proceedings of the Edinburgh Mathematical Society. Series II 20 (1976), no. 1, 73-79.

[2] J. L. Brenner, Covering theorems for finasigs. X. The group $G=\operatorname{PSL}(n, q)$ had a class $C$ such that $C C=G$, Ars Combinatoria 16 (1983), 57-67.

[3] C. M. Campbell and R. M. Thomas, On $(2, n)$-groups related to Fibonacci groups, Israel Journal of Mathematics 58 (1987), no. 3, 370-380. 
[4] H. Doostie, Non-metabelian soluble groups involving Lucas numbers, Journal of Sciences, Islamic Republic of Iran 3 (1992), 63-67.

[5] H. Doostie and A. R. Jamali, A class of deficiency zero soluble groups of derived length 4, Proceedings of the Royal Society of Edinburgh. Section A 121 (1992), no. 1-2, 163-168.

[6] V. V. Kursov, The commutant of the general linear group over a field, Doklady Akademii Nauk BSSR 23 (1979), no. 10, 869-871, 955.

[7] _ Commutators in real Chevalley groups, Doklady Akademii Nauk BSSR 28 (1984), no. 3, 208-210.

[8] O. Ore, Some remarks on commutators, Proceedings of the American Mathematical Society 2 (1951), 307-314.

[9] R. C. Thompson, Commutators in the special and general linear groups, Transactions of the American Mathematical Society 101 (1961), 16-33.

[10] H. Q. Zhang and H. You, Commutators in symplectic unimodular groups, Chinese Annals of Mathematics. Series A 12 (1991), no. 2, 238-248.

H. Doostie: Mathematics Department, Teacher Training University, 49 Mofateh Avenue, Tehran 15614, Iran

E-mail address: Doostih@saba.tmu.ac.ir

P. P. Campbell: Institute of Mathematics, St. Andrews University, St. Andrews, KY16 9SS,

Scotland, UK

E-mail address: peterc@st-andrews.ac.uk 


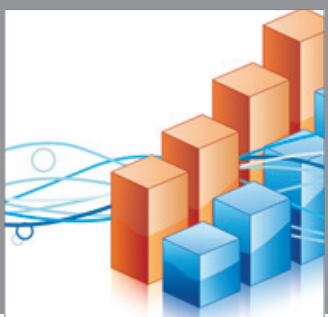

Advances in

Operations Research

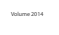

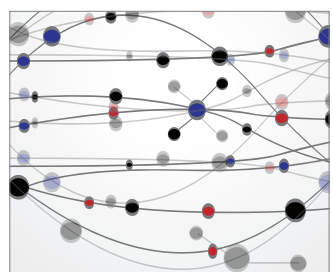

\section{The Scientific} World Journal
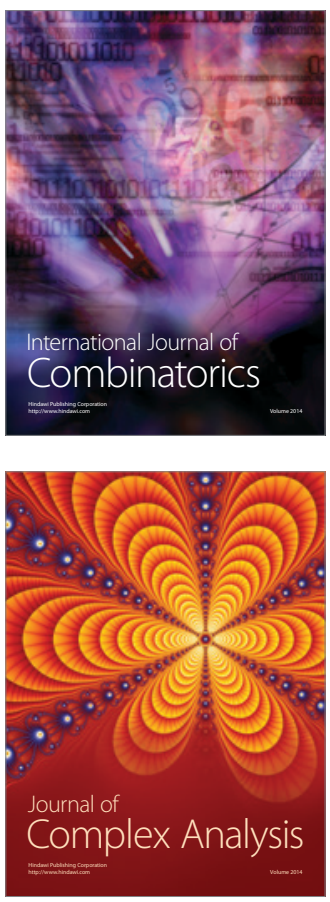

International Journal of

Mathematics and

Mathematical

Sciences
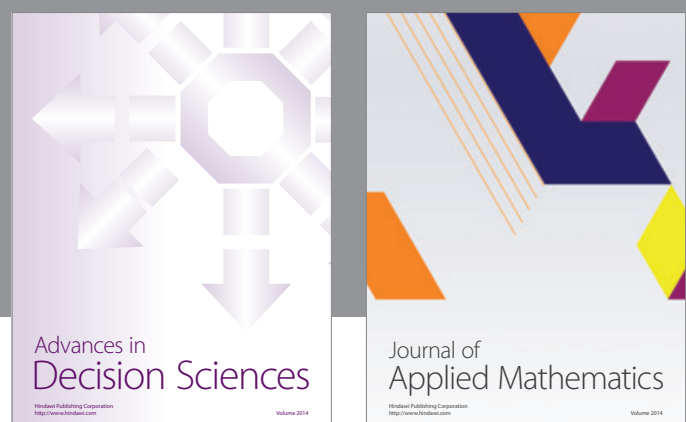

Journal of

Applied Mathematics
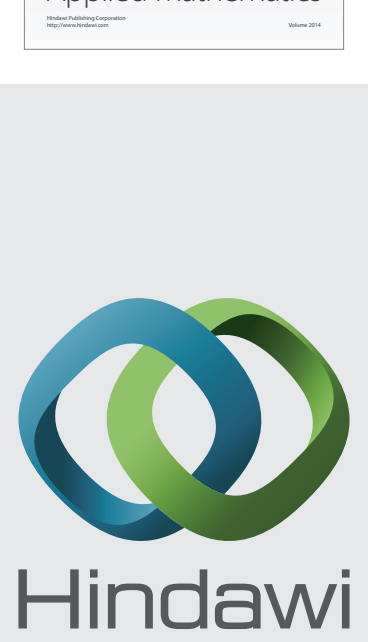

Submit your manuscripts at http://www.hindawi.com
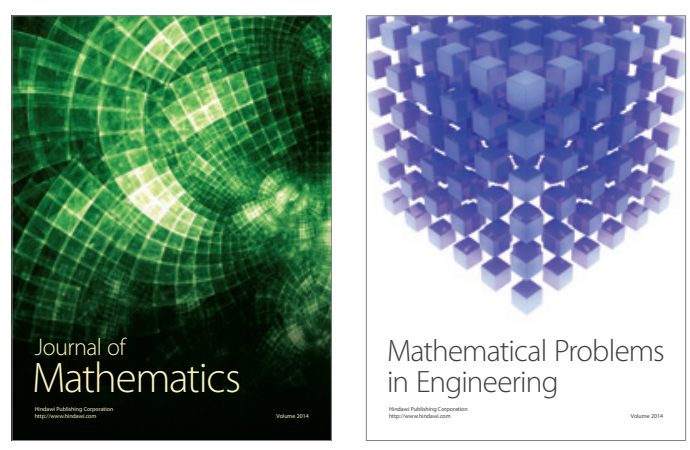

Mathematical Problems in Engineering
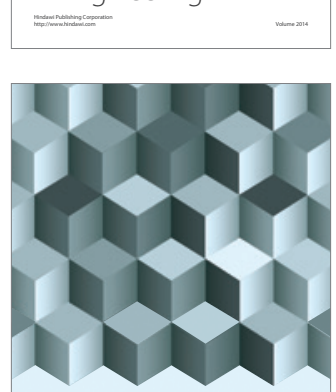

Journal of

Function Spaces
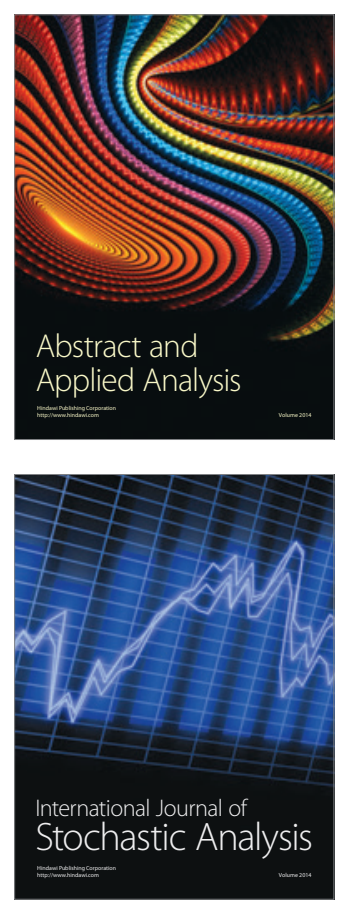

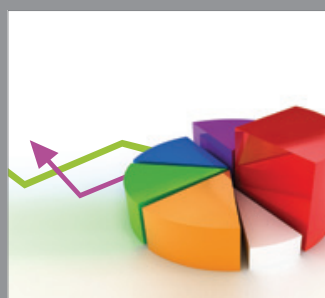

ournal of

Probability and Statistics

Promensencen
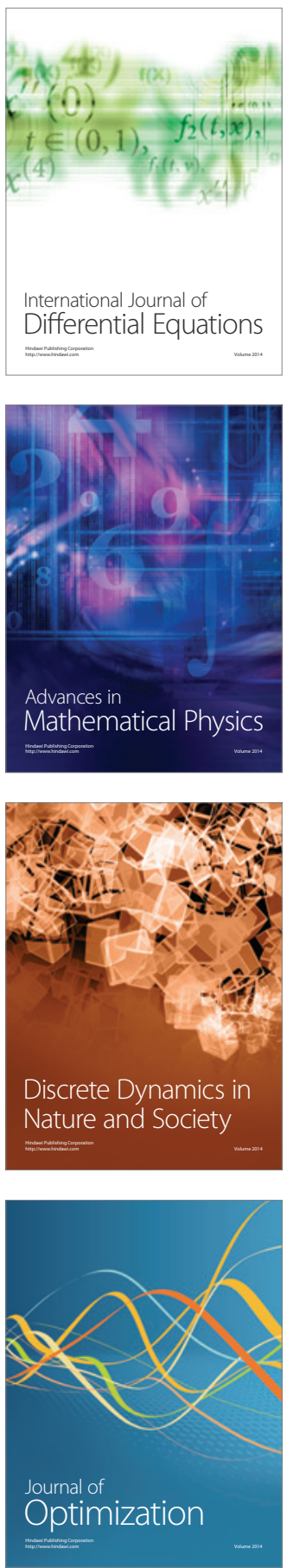